\title{
Tramadol is an Opioid
}

Bosse et al. incorrectly described tramadol as "a non-opioid analgesic". Tramadol is an oral analgesic, which stems from both norepinephrine and serotonin reuptake inhibition and direct $\mu$-receptor agonism [1]. In fact, metabolism by CYP3A4 yields the active metabolite, N-desmethyltramadol, which demonstrates greater $\mu$-opioid receptor affinity than tramadol itself [2]. The reason why this should be clarified is the misconception that tramadol is not an opioid, and, therefore, can be administered without concern for toxicity or abuse compared to other opiates or opioids. Admittedly, the incidence of abuse is relatively low but it is not nonexistent [3]. This misconception has unfortunately been perpetuated throughout different fields of medicine, from primary care physician to specialist. Moreover, the potential of drug-drug interactions and seizures are also often overlooked due to the preconceived notion of tramadol's benign nature. Lastly, even patients themselves are often unaware of the fact that tramadol is an opioid medication when they were informed by their prescribing physicians that in fact it was not [4].

Tramadol's problematic issues are discussed on a nearly daily basis in the emergency department and hospital wards often much to the surprise of medical students, residents, fellows and attending physicians. It is our responsibility as medical toxicologists- experts in the field of medication toxicity, interactions and side-effects - to properly identify medications and to instill this knowledge not only to our colleagues, but our patients as well.
Kenneth D. Katz, MD

Chief, Division of Medical Toxicology

Department of Emergency Medicine, University of Pittsburgh Medical Center

Presbyterian Hospital

Pittsburgh, PA

Katzkd@upmc.edu

\section{REFERENCES}

1. C. Hernandez-Lopez, L. Martinez-Farnos, D. Karhu, PerezCampos T, Rovira S, Encina G. Comparative Bioavailability Between Two Tramadol Once-DailyOral Formulations. Methods Find Exp Clin Pharmacol. 2006;28(6):373-378

2. Curry SC, Watts DJ, Katz KD, Bilkin D, Bukaveckas BL. The effect of single-dose tramadol on oxycodone clearance. J Emerg Med. 2007;33(4):407-11.

3. Epstein DH, Preston KL and Jasinski DR. Abuse liability, behavioral pharmacology, and physical-dependence potential of opioids in humans and laboratory animals: Lessons from tramadol. Biological Psychology. 2006;73(1):90-99.

4. R. B. Raffa PhD (2008) Basic pharmacology relevant to drug abuse assessment: tramadol as example. J Clinical Pharmacy and Therapeutics. 2008;33(2):101-108. 